TRANSACTIONS OF THE

AMERICAN MATHEMATICAL SOCIETY

Volume 357, Number 7 , Pages 2753-2770

S 0002-9947(04)03552-

Article electronically published on September 23, 2004

\title{
HOMOTOPICAL LOCALIZATIONS OF MODULE SPECTRA
}

\author{
CARLES CASACUBERTA AND JAVIER J. GUTIÉRREZ
}

\begin{abstract}
We prove that stable $f$-localizations (where $f$ is any map of spectra) preserve ring spectrum structures and module spectrum structures, under suitable hypotheses, and we use this fact to describe all possible localizations of the integral Eilenberg-Mac Lane spectrum $H \mathbb{Z}$. As a consequence of this study, we infer that localizations of stable GEMs are stable GEMs, and it also follows that there is a proper class of nonequivalent stable localizations.
\end{abstract}

\section{INTRODUCTION}

A spectrum is called a stable GEM if it is homotopy equivalent to a wedge

$$
\bigvee_{k \in \mathbb{Z}} \Sigma^{k} H A_{k}
$$

where each $A_{k}$ is an abelian group and $H A_{k}$ denotes an Eilenberg-Mac Lane spectrum with $\pi_{0}\left(H A_{k}\right) \cong A_{k}$ and $\pi_{i}\left(H A_{k}\right)=0$ if $i \neq 0$. A spectrum is a stable GEM if and only if it admits an $H \mathbb{Z}$-module structure, where $H \mathbb{Z}$ is viewed as a ring spectrum in the usual way. Equivalently, $X$ is a stable GEM if and only if $X$ is a homotopy retract of $H \mathbb{Z} \wedge X$; more details about these claims are given in Section 5 . Note the analogy with unstable homotopy, where a space $X$ is a GEM (i.e., a weak product of abelian Eilenberg-Mac Lane spaces) if and only if it is a homotopy retract of the infinite symmetric product $\mathrm{SP}^{\infty} X$, a space whose homotopy groups are the integral homology groups of $X$.

Dror Farjoun Dro96], and later Badzioch Bad01, with different methods, proved that unstable $f$-localizations send GEMs to GEMs. In this article we prove that the same result is true in stable homotopy, by developing further certain ideas used by Bousfield in [Bou96] and [Bou99].

For this, one can choose to work in any suitable simplicial model category $\mathcal{S}$ whose homotopy category is equivalent to the homotopy category of CW-spectra. In order to ensure the existence of $f$-localizations, we ask that $\mathcal{S}$ be left proper, cofibrantly generated, and locally presentable. The Bousfield-Friedlander model category [BF78] or the model category of symmetric spectra built from simplicial sets [HSS00] satisfy these conditions. However, most of our arguments are homotopic in nature and make no use of any specific models for spectra.

Received by the editors May 1, 2002 and, in revised form, November 3, 2003.

2000 Mathematics Subject Classification. Primary 55P42, 55P43, 55P60.

Key words and phrases. Localization, ring spectrum, module spectrum, stable GEM.

The authors were supported by MCyT grants PB97-0202, BFM2001-2031, and FP98 16587447. 
Homotopical localization with respect to a map $f: A \rightarrow B$ of cofibrant spectra ( $f$-localization, for short) is a functor $L_{f}$ from $\mathcal{S}$ to itself, which sends weak equivalences to weak equivalences, is idempotent in the homotopy category and takes values in the subcategory of fibrant spectra $X$ such that the map of simplicial sets

$$
\operatorname{Map}(f, X): \operatorname{Map}(B, X) \longrightarrow \operatorname{Map}(A, X)
$$

is a weak equivalence. All known forms of localizations in stable homotopy are $f$-localizations for suitable choices of the map $f$. Among these, homological localizations commute with the suspension operator. It is crucial to emphasize that not all $f$-localizations commute with suspension; we give necessary and sufficient conditions in Section 2.

We prove that if $f$ is any map of spectra and $E$ is a ring spectrum (i.e., a monoid in the homotopy category), then $L_{f} E$ is also a ring spectrum and the localization map $E \rightarrow L_{f} E$ is a ring map, if we assume either that both $E$ and $L_{f} E$ are connective or that $L_{f}$ commutes with suspension. If $E$ is commutative, then $L_{f} E$ is also commutative. Similarly, if $M$ is an $E$-module spectrum, then so is $L_{f} M$, and the localization map $M \rightarrow L_{f} M$ is an $E$-module map, provided that $E$ is connective or $L_{f}$ commutes with suspension. (A spectrum $E$ is connective if its homotopy groups $\pi_{i}(E)$ vanish for $i<0$.)

It will be shown elsewhere that a stronger result holds, namely that, if the model category $\mathcal{S}$ is closed symmetric monoidal and $E$ is a monoid in $\mathcal{S}$, then $L_{f} E$ is homotopy equivalent to a monoid in $\mathcal{S}$, if we assume either that both $E$ and $L_{f} E$ are connective or that $L_{f}$ commutes with suspension. The same is true for commutative monoids. This is proved in the special case of homological localizations in Chapter VIII of [EKMM97.

It follows that $f$-localizations send $H \mathbb{Z}$-modules to $H \mathbb{Z}$-modules; that is, the class of stable GEMs is preserved by $f$-localizations. We show that, in fact, for every abelian group $G$,

$$
L_{f} H G \simeq H A \vee \Sigma H B
$$

for certain abelian groups $A$ and $B$. When $G=\mathbb{Z}$, we show that $B=0$ and the group $A$ is a commutative ring with 1 such that $\operatorname{Hom}(A, A) \cong A$ via $\varphi \mapsto \varphi(1)$. Rings $A$ with this property were called rigid in [CRT00. It was shown in DMV87. that there is a proper class of nonisomorphic rigid rings, and it is easy to see that for every rigid ring $A$ there is a map $f$ such that $L_{f} H \mathbb{Z} \simeq H A$. Remarkably, this shows that there is a proper class of nonequivalent $f$-localizations in the stable homotopy category. In contrast, recall from [DP01] that there is only a set of nonequivalent homological localizations. We do not know whether there is a proper class or a set of nonequivalent $f$-localizations in the stable homotopy category that commute with suspension. This question seems to be closely related to other unanswered questions about Bousfield classes, cohomological localizations, and localizing subcategories, some of which appear in the work of Hovey, Palmieri and Strickland, e.g. [Hov95, HPS97, Str04.

A more conceptual explanation of the fact that, for every abelian group $G$, the spectrum $L_{f} H G$ has at most two nonzero homotopy groups is that the homotopy category of $H R$-modules is equivalent to the homotopy category of ( $\mathbb{Z}$-graded) chain complexes of $R$-modules, where $R$ is any ring; see [EKMM97, IV.2]. However, this holds for strict $H R$-modules; that is, modules over $H R$ in a closed symmetric monoidal category of spectra. For certain rings, including $R=\mathbb{Z}$, the distinction 
between strict $H R$-modules and homotopy $H R$-modules (i.e., $H R$-module spectra in the homotopy category) is unessential, but for other rings it is crucial. This phenomenon is discussed in Gut03.

As an application of our results, we show that if $E=K$ (complex $K$-theory) or $E=E(n)$ (the Johnson-Wilson spectrum) for some $n$, then the $E$-localization of the sphere spectrum $S$ has the following homology groups:

$$
H_{0}\left(L_{E} S\right) \cong \mathbb{Q}, \quad H_{i}\left(L_{E} S\right)=0 \quad \text { for } i \neq 0 .
$$

A similar result (with possibly other subrings of the rationals) holds for every smashing localization, in the sense of Rav84. It follows as an easy consequence that, if $L$ is a smashing localization such that $H_{0}(L S) \cong \mathbb{Q}$, then either $L$ is ordinary $H \mathbb{Q}$-localization, or $L S$ is unbounded.

\section{Localization of SPECTRA With RESPECT TO A MAP}

The notion of $f$-localization can be formulated in any category $\mathcal{S}$ with a simplicial model structure; see GJ99] or [Hir03 for further details about methods and terminology. Given a map $f: A \rightarrow B$ between cofibrant objects in such a category, an object $X$ is called $f$-local if it is fibrant and the induced map of simplicial sets

$$
\operatorname{Map}(f, X): \operatorname{Map}(B, X) \longrightarrow \operatorname{Map}(A, X)
$$

is a weak equivalence. We label the following standard properties for later use.

Lemma 2.1. If $X$ is $f$-local and $Y \rightarrow X$ is a map with a left homotopy inverse, then $Y$ is $f$-local.

Proof. Factor $Y \rightarrow X$ into a cofibration $Y \rightarrow Z$ followed by a trivial fibration $Z \rightarrow X$. Then $Z$ is $f$-local and $Y$ is a retract of $Z$. It follows, as in Hir03, 1.2.5], that $\operatorname{Map}(f, Y): \operatorname{Map}(B, Y) \rightarrow \operatorname{Map}(A, Y)$ is a retract of $\operatorname{Map}(f, Z)$, and hence it is a weak equivalence.

Lemma 2.2. Every homotopy limit of $f$-local objects is $f$-local.

Proof. If $I$ is a small category and $D: I \rightarrow \mathcal{S}$ is a diagram in $\mathcal{S}$ taking values in the subcategory of $f$-local objects, then

$$
\begin{aligned}
\operatorname{Map}\left(B, \operatorname{holim}_{I} D\right) & \cong \operatorname{holim}_{I} \operatorname{Map}(B, D) \\
& \simeq \operatorname{holim}_{I} \operatorname{Map}(A, D) \cong \operatorname{Map}\left(A, \operatorname{holim}_{I} D\right),
\end{aligned}
$$

where $\operatorname{Map}(B, D)$ and $\operatorname{Map}(A, D)$ denote the corresponding diagrams of simplicial sets indexed by $I$.

A map $g: X \rightarrow Y$ is an $f$-equivalence if there is a cofibrant approximation $\tilde{g}: \tilde{X} \rightarrow \tilde{Y}$ such that

$$
\operatorname{Map}(\tilde{g}, E): \operatorname{Map}(\tilde{Y}, E) \longrightarrow \operatorname{Map}(\tilde{X}, E)
$$

is a weak equivalence for every $f$-local object $E$. Similarly as in Lemmas 2.1 and 2.2, every homotopy retract of an $f$-equivalence is an $f$-equivalence, and every homotopy colimit of $f$-equivalences is an $f$-equivalence. In particular, every coproduct of $f$ equivalences is an $f$-equivalence.

An $f$-localization of an object $X$ is an $f$-equivalence $l: X \rightarrow L_{f} X$, where $L_{f} X$ is $f$-local. Such a map $l$ is initial in the homotopy category HoS among maps from $X$ to $f$-local objects, and it is terminal in HoS among $f$-equivalences with domain $X$. 
Each of these two universal properties ensures that, if an $f$-localization of $X$ exists, then it is unique up to homotopy.

The existence of $f$-localization for every map $f$ and all objects $X$ is guaranteed if the simplicial model category $\mathcal{S}$ satisfies certain assumptions. Specifically, the following result is proved as indicated in Bou77. or in Hir03. The notion of a locally presentable category was defined in GU71]. The definitions of the terms cofibrantly generated and left proper can be found in [Hir03.

Theorem 2.3. Let $\mathcal{S}$ be any locally presentable, cofibrantly generated, left proper, simplicial model category. Then for every map $f$ there exists a functor $L_{f}$ on $\mathcal{S}$ with a natural transformation $\mathrm{Id} \rightarrow L_{f}$ yielding an $f$-localization for all objects.

(Such a functor need not be unique on $\mathcal{S}$, but it is unique up to homotopy, and a standard construction provides canonical models.) The Bousfield-Friedlander category of spectra BF78 or the category of symmetric spectra built from simplicial sets HSS00 satisfies the assumptions stated in Theorem 2.3. Moreover, for $X$ cofibrant and $Y$ fibrant, the simplicial set $\operatorname{Map}(X, Y)$ is an infinite loop space and, for any choice of basepoint, its homotopy groups satisfy

$$
\pi_{k}(\operatorname{Map}(X, Y), *) \cong\left[\Sigma^{k} X, Y\right] \cong \pi_{k}(F(X, Y)) \text { for } k>0,
$$

where $F(X, Y)$ denotes the function spectrum from $X$ to $Y$. Thus, the homotopy groups of the simplicial set $\operatorname{Map}(X, Y)$ are isomorphic to those of the connective cover $F^{c}(X, Y)$ of the function spectrum from $X$ to $Y$.

From now on, we will work with (derived) function spectra in the homotopy category, and omit any further reference to the chosen model category. Thus, for a map $f: A \rightarrow B$ in HoS, a spectrum $E$ is $f$-local if and only if the induced map of connective covers of function spectra

$$
F^{c}(B, E) \longrightarrow F^{c}(A, E)
$$

is an equivalence, i.e., induces isomorphisms of all homotopy groups. Likewise, a map $g: X \rightarrow Y$ in HoS is an $f$-equivalence if and only if the induced map

$$
F^{c}(Y, E) \longrightarrow F^{c}(X, E)
$$

is an equivalence for every $f$-local spectrum $E$. These are indeed the definitions adopted in Bou96] and Bou99.

Proposition 2.4. Let $f$ be any map.

(a) If $E$ is $f$-local, then $\Sigma^{-k} E$ is also $f$-local for $k \geq 0$.

(b) If $g: X \rightarrow Y$ is an $f$-equivalence, then $\Sigma^{k} g$ is also an $f$-equivalence for $k \geq 0$.

Proof. Part (a) is a special case of Lemma 2.2. A more direct argument can however be given as follows. If $E$ is $f$-local, then $f$ induces an equivalence

$$
F^{c}\left(B, \Sigma^{-k} E\right) \simeq F^{c}\left(A, \Sigma^{-k} E\right) \text { for } k \geq 0,
$$

since $\pi_{i}\left(F^{c}\left(B, \Sigma^{-k} E\right)\right) \cong \pi_{i+k}\left(F^{c}(B, E)\right)$ if $i \geq 0$ and $k \geq 0$. The proof of part (b) is similar.

Since $F^{c}(B, E) \simeq F^{c}\left(\Sigma^{k} B, \Sigma^{k} E\right)$ for all $B, E$ and $k \in \mathbb{Z}$, we may also infer that if $E$ is $f$-local then $\Sigma^{k} E$ is $\Sigma^{k} f$-local for every $k \in \mathbb{Z}$, and similarly for $f$-equivalences. From this fact we deduce the following result. 
Proposition 2.5. For every map $f$ and every spectrum $X$ there is an equivalence

$$
L_{f} \Sigma^{-k} X \simeq \Sigma^{-k} L_{\Sigma^{k} f} X \quad \text { for all } k \in \mathbb{Z} .
$$

Proof. Since $l: X \rightarrow L_{\Sigma^{k} f} X$ is a $\Sigma^{k} f$-equivalence, $\Sigma^{-k} l$ is an $f$-equivalence. Moreover, $\Sigma^{-k} L_{\Sigma^{k} f} X$ is $f$-local since $L_{\Sigma^{k} f} X$ is $\Sigma^{k} f$-local, so our claim follows.

This is to be compared with the expression $L_{f} \Omega^{k} X \simeq \Omega^{k} L_{\Sigma^{k} f} X$ for spaces, which was proved in Dro96.

Proposition 2.6. Let $X \rightarrow Y \rightarrow Z$ be a cofibre sequence of spectra and let $f$ be any map. If $L_{f} X$ is contractible, then the map $Y \rightarrow Z$ is an $f$-equivalence.

Proof. Consider the long exact sequence of abelian groups

$$
\cdots \rightarrow[\Sigma X, E] \rightarrow[Z, E] \rightarrow[Y, E] \rightarrow[X, E] \rightarrow \cdots
$$

for a spectrum $E$. If $E$ is $f$-local, then $\left[\Sigma^{k} X, E\right]=0$ for $k \geq 0$, by part (b) of Proposition [2.4. Therefore, $[Z, E] \cong[Y, E]$ for all $f$-local spectra $E$, and this implies that the map $Y \rightarrow Z$ is an $f$-equivalence.

For arbitrary spectra $X$ and $Y$ and any map $f$, there is a natural equivalence

$$
L_{f} X \vee L_{f} Y \simeq L_{f}(X \vee Y),
$$

since the coproduct $X \vee Y \rightarrow L_{f} X \vee L_{f} Y$ is an $f$-equivalence and $L_{f} X \vee L_{f} Y$ is a product of $f$-local spectra, hence $f$-local (the same argument is valid for any finite wedge). Thus, $L_{f}$ is an additive functor on HoS.

Localization with respect to a map of the form $f: A \rightarrow 0$ is called A-nullification, and it is denoted by $P_{A}$ instead of $L_{f}$. The corresponding local spectra are called $A$-null. Thus, a spectrum $X$ is $A$-null if and only if $F^{c}(A, X) \simeq 0$. For every map $f$ there is a natural transformation $P_{C} \rightarrow L_{f}$, where $C$ is the cofibre of $f$. This follows from the fact that every $f$-local spectrum is $C$-null.

As a consequence of Proposition 2.4 for every $X$ there is a natural map

$$
\Sigma L_{f} X \longrightarrow L_{f} \Sigma X \text {. }
$$

We say that $L_{f}$ commutes with suspension if this natural map is an equivalence for all $X$. Thus, $L_{f}$ commutes with suspension if and only if $\Sigma L_{f} X \simeq L_{f} \Sigma X$, since the latter implies that $\Sigma L_{f} X$ is $f$-local, and the map (2.1) is then an $f$-equivalence between $f$-local spectra, hence an equivalence.

As we next show in detail, $L_{f}$ commutes with suspension if and only if it is an exact functor on the stable homotopy category, or a triangulated functor in the sense of Neeman [Nee01.

Theorem 2.7. Let $f: A \rightarrow B$ be a map of spectra. Then the following statements are equivalent:

(i) $\Sigma L_{f} X \simeq L_{f} \Sigma X$ for every spectrum $X$.

(ii) $\Sigma^{k} L_{f} X \simeq L_{f} \Sigma^{k} X$ for every spectrum $X$ and every $k \in \mathbb{Z}$.

(iii) If $E$ is any $f$-local spectrum, then $\Sigma^{k} E$ is $f$-local for all $k \in \mathbb{Z}$.

(iv) If $g$ is any $f$-equivalence, then $\Sigma^{k} g$ is an $f$-equivalence for all $k \in \mathbb{Z}$.

(v) $L_{f} X \simeq L_{\Sigma^{k} f} X$ for every spectrum $X$ and every $k \in \mathbb{Z}$.

(vi) The map $F(B, E) \rightarrow F(A, E)$ induced by $f$ is an equivalence for every $f$-local spectrum $E$.

(vii) If $E$ is $f$-local and $X$ is any spectrum, then $F(X, E)$ is $f$-local. 
(viii) If $g: X \rightarrow Y$ and $h: M \rightarrow N$ are $f$-equivalences, then $g \wedge h: X \wedge M \rightarrow Y \wedge N$ is also an $f$-equivalence.

(ix) If $X \rightarrow Y \rightarrow Z$ is any cofibre sequence of spectra, then $L_{f} X \rightarrow L_{f} Y \rightarrow L_{f} Z$ is also a cofibre sequence.

Proof. Clearly (i) and (ii) are equivalent, and (ii) implies (iii). Note that (iii) implies that $\Sigma L_{f} X$ is $f$-local for all $X$, and hence (i) holds because (2.1) is an $f$-equivalence. It is straightforward that (iii) and (iv) are equivalent. From (iv) it follows that, given any $k \in \mathbb{Z}$, the map $\Sigma^{k} f$ is an $f$-equivalence, and $f$ is also a $\Sigma^{k} f$-equivalence. This implies that the classes of $f$-local spectra and $\Sigma^{k} f$-local spectra coincide, thus yielding (v). Now (v) together with Proposition 2.5 implies (ii).

Statement (vi) is equivalent to the fact that $f$ induces equivalences

$$
F^{c}\left(B, \Sigma^{k} E\right) \simeq F^{c}\left(A, \Sigma^{k} E\right)
$$

for all $k \in \mathbb{Z}$, and hence it follows from (iii). To prove (vii), we have to verify that

$$
F^{c}(B, F(X, E)) \simeq F^{c}(A, F(X, E)),
$$

which is equivalent to $F^{c}(X, F(B, E)) \simeq F^{c}(X, F(A, E))$, and this follows from (vi). Statement (viii) is proved from (vii) by taking any $f$-local spectrum $E$ and observing that

$$
\begin{aligned}
F^{c}(Y \wedge N, E) \simeq F^{c}(Y, F(N, E)) \simeq F^{c}(X, F(N, E)) \\
\\
\simeq F^{c}(N, F(X, E)) \simeq F^{c}(M, F(X, E)) \simeq F^{c}(X \wedge M, E) .
\end{aligned}
$$

Now (iv) follows from (viii) by smashing $g$ with the identity of $\Sigma^{k} S$, for $k \in \mathbb{Z}$. Hence, statements (i) to (viii) are equivalent.

We next deduce (ix) from (i). Given a cofibre sequence $X \rightarrow Y \rightarrow Z$, consider the ladder

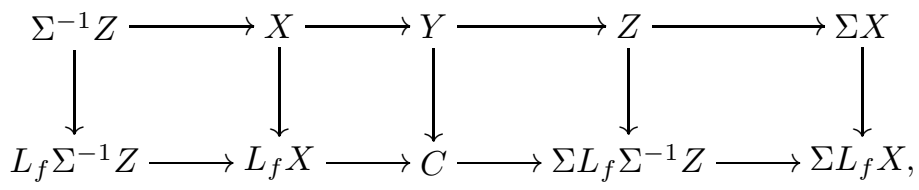

where $C$ is the cofibre of the map $L_{f} \Sigma^{-1} Z \rightarrow L_{f} X$. It follows from (i) that $\Sigma L_{f} \Sigma^{-1} Z \simeq L_{f} Z$, and $\Sigma L_{f} X \simeq L_{f} \Sigma X$. Thus, all vertical maps except perhaps $Y \rightarrow C$ are $f$-equivalences. The five-lemma implies then that $Y \rightarrow C$ is also an $f$-equivalence and that $C$ is $f$-local. Therefore $C \simeq L_{f} Y$. Finally, in order to prove that (ix) implies (i), use the cofibre sequence $X \rightarrow 0 \rightarrow \Sigma X$, for any $X$.

Statement (vi) tells us that $f$-localization commutes with suspension if and only if the class of $f$-local spectra is characterized by means of the full function spectrum $F$, instead of its connective cover $F^{c}$. We also remark that, by the following fact, every $f$-localization that commutes with suspension is a nullification.

Corollary 2.8. If a localization functor $L_{f}$ commutes with suspension, then the natural transformation $P_{C} \rightarrow L_{f}$, where $C$ is the cofibre of $f$, is an equivalence.

Proof. There are natural transformations $L_{\Sigma f} \rightarrow P_{C} \rightarrow L_{f}$, which correspond to inclusions of the respective classes of local spectra. Since the composite $L_{\Sigma f} \rightarrow L_{f}$ is an equivalence by part (v) of Theorem [2.7, the arrow $P_{C} \rightarrow L_{f}$ is also an equivalence. 
For a given map $f$, if we consider the wedge $g=\bigvee_{k \in \mathbb{Z}} \Sigma^{k} f$, then there is a natural transformation $L_{f} \rightarrow L_{g}$, and $L_{g}$ commutes with suspension, by part (v) of Theorem 2.7, since $\Sigma g \simeq g$. This natural transformation $L_{f} \rightarrow L_{g}$ is an equivalence if and only if $L_{f}$ commutes with suspension, since a spectrum is then $g$-local if and only if it is $f$-local. The same holds if we replace $g$ by any map of the form $\bigvee_{k<N} \Sigma^{k} f$, where $N$ is any integer.

\section{EXAMPles of $f$-LOCALIZATIONS}

We briefly discuss three well-known examples of localizations in the stable homotopy category, namely Postnikov sections, homological localizations and localizations at sets of primes, and display them as $f$-localizations for certain maps $f$.

3.1. Postnikov sections. The localization of a spectrum $E$ with respect to the map $f: \Sigma^{k+1} S \rightarrow 0$, where $S$ is the sphere spectrum and $k \in \mathbb{Z}$, is equivalent to the $k$-th Postnikov section $E_{(k)}$ of $E$. Recall that $\pi_{i}\left(E_{(k)}\right)=0$ for $i>k$, and that there is a map $E \rightarrow E_{(k)}$ inducing isomorphisms of homotopy groups in dimensions less than or equal to $k$. Postnikov sections do not commute with suspension. Indeed, if $\pi_{k}(E) \neq 0$, then $(\Sigma E)_{(k)} \not \Sigma E_{(k)}$.

3.2. Homological localizations. Homological localizations in stable homotopy were first constructed in [Bou79]. Let $E$ be any spectrum. Then a spectrum $X$ is called $E$-acyclic if $E_{k}(X)=0$ for all $k \in \mathbb{Z}$ or, equivalently, if $E \wedge X \simeq 0$. A map of spectra $g: X \rightarrow Y$ is an E-equivalence if the map $g_{*}: E_{k}(X) \rightarrow E_{k}(Y)$ is an isomorphism for all $k \in \mathbb{Z}$, that is, if $g$ induces an equivalence $E \wedge X \simeq E \wedge Y$. Thus, $g$ is an $E$-equivalence if and only if its cofibre is $E$-acyclic. A spectrum $Z$ is $E$-local if each $E$-equivalence $f: X \rightarrow Y$ induces an equivalence $F(Y, Z) \simeq F(X, Z)$ or, equivalently, if $F(A, Z) \simeq 0$ for each $E$-acyclic spectrum $A$.

An E-localization of a spectrum $X$ is an $E$-equivalence $X \rightarrow L_{E} X$ from $X$ to an $E$-local spectrum. E-localization is a nullification, as shown in Bou79]. We label this fact for further reference:

Theorem 3.1. Let $E$ be any spectrum. Then there are an E-acyclic spectrum $A$ and a natural equivalence $P_{A} X \simeq L_{E} X$ for every spectrum $X$.

Homological localizations clearly commute with suspension.

3.3. Localization at sets of primes. Let $G$ be any abelian group and let $M G$ denote its Moore spectrum. Thus, $(H \mathbb{Z})_{0}(M G) \cong \pi_{0}(M G) \cong G, \pi_{i}(M G)=0$ if $i<0$, and $(H \mathbb{Z})_{i}(M G)=0$ if $i \neq 0$. Here $H \mathbb{Z}$ denotes the integral EilenbergMac Lane spectrum.

Lemma 3.2. There is a natural exact sequence

$$
0 \longrightarrow \operatorname{Ext}\left(G, \pi_{k+1}(X)\right) \longrightarrow\left[\Sigma^{k} M G, X\right] \longrightarrow \operatorname{Hom}\left(G, \pi_{k}(X)\right) \longrightarrow 0
$$

for each spectrum $X$, each abelian group $G$, and all $k$, where $M G$ is the Moore spectrum associated with $G$.

Proof. Pick a free presentation of the group $G$ and use the corresponding cofibre sequence of Moore spectra; cf. Bou79, (2.2)]. 
Let $\mathbb{Z}_{P}$ denote the integers localized at a set of primes $P$ (possibly empty). For every spectrum $X$, the $P$-localization of $X$ is the natural map

$$
1 \wedge \eta: X \longrightarrow X \wedge M \mathbb{Z}_{P}
$$

where $\eta: S \rightarrow M \mathbb{Z}_{P}$ corresponds to the unit in $\mathbb{Z}_{P}=\pi_{0}\left(M \mathbb{Z}_{P}\right)$. A spectrum $X$ is $P$-local if this map is an equivalence. We shall write $X_{P}$ for $X \wedge M \mathbb{Z}_{P}$.

It follows from the definition that $P$-localization is a homological localization, namely $M \mathbb{Z}_{P}$-localization. Hence, $P$-localization commutes with suspension. We also remark that

$$
X_{P} \simeq X \wedge S_{P}
$$

for all $X$; that is, $P$-localization is smashing (see the end of Section 5).

For each $k \in \mathbb{Z}$ we have

$$
\pi_{k}\left(X_{P}\right) \cong \pi_{k}(X) \otimes \pi_{0}\left(M \mathbb{Z}_{P}\right) \cong \pi_{k}(X) \otimes \mathbb{Z}_{P},
$$

and from this fact it follows that, if $E$ is any spectrum, then

$$
E_{k}\left(X_{P}\right) \cong \pi_{k}\left(E \wedge X \wedge M \mathbb{Z}_{P}\right) \cong E_{k}(X) \otimes \mathbb{Z}_{P}
$$

for all $X$. Hence, $P$-localization of spectra induces $P$-localization of their homotopy and homology groups. If we consider cohomology, then Lemma 3.2 yields

$$
\begin{aligned}
& E^{k}\left(X_{P}\right) \cong\left[X \wedge M \mathbb{Z}_{P}, \Sigma^{k} E\right] \cong\left[M \mathbb{Z}_{P}, F\left(X, \Sigma^{k} E\right)\right] \\
& \cong \operatorname{Hom}\left(\mathbb{Z}_{P}, E^{k}(X)\right) \oplus \operatorname{Ext}\left(\mathbb{Z}_{P}, E^{k-1}(X)\right) .
\end{aligned}
$$

An explicit map $f$ such that $L_{f} X \simeq X_{P}$ for all $X$ can be displayed as follows.

Proposition 3.3. Let $P$ be a set of primes and let $g: \bigvee_{q \notin P} S \rightarrow \bigvee_{q \notin P} S$ be a wedge of maps inducing multiplication by $q$ in $\pi_{0}(S)$ for each prime $q$ not in $P$, and let $f=\bigvee_{n<0} \Sigma^{n} g$. Then $L_{f} X \simeq X \wedge M \mathbb{Z}_{P}$ for all $X$.

Proof. The map $f$ has been chosen so that $f$-local spectra are precisely those spectra whose homotopy groups are $\mathbb{Z}_{P}$-modules. For a spectrum $X$, the $P$-localization map $X \rightarrow X_{P}$ is an equivalence if and only if the homotopy groups of $X$ are $\mathbb{Z}_{P}$-modules, by (3.1). Thus we conclude the proof by recalling that two localization functors with the same class of local spectra are necessarily equivalent.

Note that $P$-localization is also equivalent to nullification with respect to the cofibre of $f$, which is a wedge of Moore spectra, namely

$$
\bigvee_{q \notin P, n<0} \Sigma^{n} M \mathbb{Z} / q
$$

One could also consider the effect of nullification with respect to $M=\bigvee_{q \notin P} M \mathbb{Z} / q$ (without the desuspensions). The resulting functor does not commute with suspension and does not change the homotopy groups of spectra in negative dimensions, since $M$ is connective (see Remark 4.6). From Lemma 3.2 it follows that the $M$-null spectra are the spectra $X$ such that $\pi_{n}(X)$ is a $\mathbb{Z}_{P}$-module for $n>0$ and $\pi_{0}(X)$ is $P^{\prime}$-torsion free, where $P^{\prime}$ denotes the complement of $P$. Hence, nullification with respect to $M$ kills the $P^{\prime}$-torsion on $\pi_{0}$ and $P$-localizes the homotopy groups in positive dimensions. In fact there is a sequence of localization functors,

$$
\cdots \rightarrow L_{\Sigma g} \rightarrow P_{M} \rightarrow L_{g} \rightarrow P_{\Sigma^{-1} M} \rightarrow L_{\Sigma^{-1} g} \rightarrow P_{\Sigma^{-2} M} \rightarrow \cdots
$$

converging to the identity in one direction and to $P$-localization in the other direction, similarly as in CR97. 


\section{LOCALIZATIONS OF RING SPECTRA AND MODULE SPECTRA}

We recall the definition of ring spectra and module spectra in the homotopy category, as in Ada74]. A spectrum $E$ is called a ring spectrum if it is equipped with two maps $\mu: E \wedge E \rightarrow E$ and $\eta: S \rightarrow E$ such that the following diagrams commute up to homotopy:
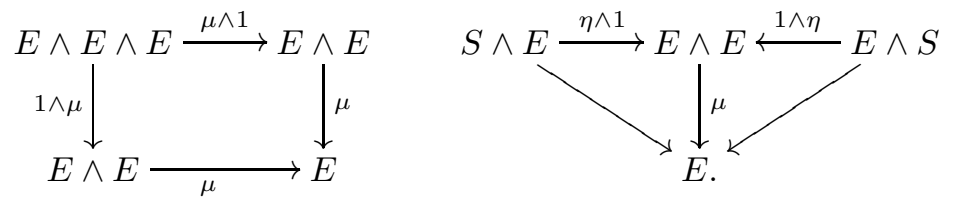

It is said that $E$ is commutative if $\mu \circ \tau \simeq \mu$, where $\tau: E \wedge E \rightarrow E \wedge E$ is the twist map. A spectrum $M$ is called a module spectrum over a ring spectrum $E$, or an $E$-module, if it is equipped with a map $m: E \wedge M \rightarrow M$ such that the following diagrams commute up to homotopy:
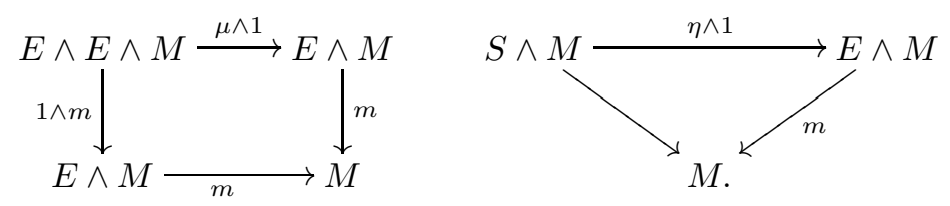

Every ring spectrum $E$ is an $E$-module spectrum with $m=\mu$.

A ring map between ring spectra $(E, \mu, \eta)$ and $\left(E^{\prime}, \mu^{\prime}, \eta^{\prime}\right)$ is a map $f: E \rightarrow E^{\prime}$ such that $f \circ \mu \simeq \mu^{\prime} \circ(f \wedge f)$ and $f \circ \eta \simeq \eta^{\prime}$. An E-module map is defined similarly.

If $R$ is a ring with unit and $M$ is a left $R$-module, then the Eilenberg-Mac Lane spectrum $H R$ is a ring spectrum, and $H M$ is a module spectrum over $H R$. The structure maps on $H R$ and $H M$ come from the product $R \otimes R \rightarrow R$ and the unit $\mathbb{Z} \rightarrow R$ in the ring $R$, and from the structure homomorphism $R \otimes M \rightarrow M$ of $M$ as an $R$-module. In particular, $H R$ is an $H \mathbb{Z}$-module for every $\operatorname{ring} R$. Moreover, every $H R$-module is an $H \mathbb{Z}$-module via the map $H \mathbb{Z} \rightarrow H R$ corresponding to the unit $\mathbb{Z} \rightarrow R$.

Remark 4.1. If $M$ is an $E$-module spectrum, then, for every spectrum $X$, the graded abelian group $\left[\Sigma^{*} X, M\right]$ is a $\pi_{*}(E)$-module, as follows. For every map $\alpha: \Sigma^{i} S \rightarrow E$ and every map $f: \Sigma^{j} X \rightarrow M$ we obtain another map $\Sigma^{i+j} X \rightarrow M$ by smashing $\alpha$ with $f$ and composing with the $E$-module structure map:

$$
\Sigma^{i+j} X \simeq \Sigma^{i} S \wedge \Sigma^{j} X \stackrel{\alpha \wedge f}{\longrightarrow} E \wedge M \stackrel{m}{\longrightarrow} M .
$$

In particular, if $M$ is an $H R$-module spectrum, then $\pi_{n}(M)$ is an $R$-module for every $n$.

As we next prove, in the case of $f$-localization functors that commute with suspension, the $f$-localizations of ring spectra or module spectra acquire a compatible ring structure or module structure. In the rest of this section, we assume that $f$ is a fixed map of spectra, and write $L$ instead of $L_{f}$.

Theorem 4.2. If the localization functor $L$ commutes with suspension, then the following hold:

(i) If $E$ is a ring spectrum, then the spectrum LE has a unique ring spectrum structure such that the localization map $l_{E}: E \rightarrow L E$ is a ring map. If $E$ is commutative, then LE is also commutative. 
(ii) If $M$ is an E-module, then the spectrum $L M$ has a unique E-module structure such that the localization map $l_{M}: M \rightarrow L M$ is an E-module map. Moreover, LM admits a unique LE-module structure extending the Emodule structure.

Proof. For the first part we need to construct a product $\bar{\mu}$ and a unit $\bar{\eta}$ on $L E$. Let $\mu$ and $\eta$ be the product and the unit of the ring spectrum $E$, respectively. We have an equivalence $F(E, L E) \simeq F(L E, L E)$ because $L E$ is local and the functor $L$ commutes with suspension by assumption. Then,

$$
\begin{aligned}
{[E \wedge E, L E] \cong[E, F(E, L E)] \cong[E, F(L E, L E)] \cong[E \wedge L E, L E] } \\
\cong[L E, F(E, L E)] \cong[L E, F(L E, L E)] \cong[L E \wedge L E, L E] .
\end{aligned}
$$

Hence, the product $\mu: E \wedge E \rightarrow E$ extends to a unique map $\bar{\mu}: L E \wedge L E \rightarrow L E$ rendering homotopy commutative the diagram

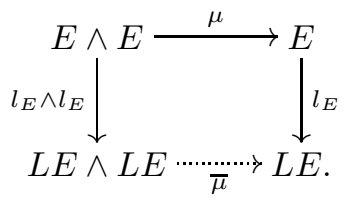

We define the unit $\bar{\eta}$ as the composition $l_{E} \circ \eta$. The commutativity of the diagrams for $\bar{\mu}$ and $\bar{\eta}$ follows from the commutativity of the diagrams for $\mu$ and $\eta$ and the universal property of $L$ (using part (viii) of Theorem [2.7).

The commutativity of $L E$ when $E$ is commutative and all the statements in part (ii) are proved in the same way.

As we next show, localization functors not commuting with suspension need not preserve ring structures nor module structures in general. The following lemma is useful to prove that certain spectra fail to be ring spectra or module spectra.

Lemma 4.3. Let $E$ and $F$ be ring spectra, and let $M$ be an E-module spectrum. If $F_{0}(E)=0$, then $F_{i}(M)=0$ for all $i \in \mathbb{Z}$.

Proof. Since $E$ and $F$ are ring spectra, $E \wedge F$ is also a ring spectrum. The assumption $F_{0}(E)=0$ says that the unit map $S \rightarrow E \wedge F$ is null, and this implies that $E \wedge F \simeq 0$. If $M$ is an $E$-module, then the composite

$$
S \wedge M \longrightarrow E \wedge M \longrightarrow M,
$$

where the first arrow comes from the unit of $E$ and the second arrow is the structure map of $M$ as an $E$-module, is an equivalence. By smashing this sequence with $F$, we find that $F \wedge M \simeq 0$, as claimed.

Example 4.4. Given a natural number $n$ and a fixed prime $p$, let $K(n)$ denote the ring spectrum corresponding to $n$th Morava $K$-theory. If we consider its 0 th Postnikov section $K(n)_{(0)}$, then, as observed in p. 95 of $\underline{\operatorname{Rud} 98},(H \mathbb{Z} / p)_{0}\left(K(n)_{(0)}\right)=0$ and $(H \mathbb{Z} / p)_{i}\left(K(n)_{(0)}\right) \neq 0$ for some $i>0$. This implies that $K(n)_{(0)}$ cannot be a ring spectrum, by Lemma 4.3. We give another approach with further details to this example, in view of its relevance. There is a cofibre sequence

$$
\Sigma^{d} k(n) \longrightarrow K(n) \longrightarrow K(n)_{(0)},
$$

where $d=2\left(p^{n}-1\right)$ and $k(n)$ denotes as usual the connective cover of $K(n)$. Suppose that $K(n)_{(0)}$ were a ring spectrum. Then $K(n)_{(0)} \wedge H \mathbb{Z} / p$ would also be 
a ring spectrum. Since $K(n) \wedge H \mathbb{Z} / p \simeq 0$, it follows that $(H \mathbb{Z} / p)_{0}\left(K(n)_{(0)}\right)=0$. Therefore the unit $S \rightarrow K(n)_{(0)} \wedge H \mathbb{Z} / p$ would be null, and this would imply that $K(n)_{(0)} \wedge H \mathbb{Z} / p \simeq 0$. But this is false, since the $\bmod p$ cohomology of $k(n)$ is a nonzero quotient of the Steenrod algebra; see, e.g., p. 545 of Rud98.

A similar argument, now considering $K(n)$ as a $K(n)$-module, and using that $(H \mathbb{Z} / p)_{0}(K(n))=0$ and Lemma 4.3 shows that $K(n)_{(0)}$ is not a $K(n)$-module.

This difficulty can be repaired by imposing suitable connectivity conditions. The following result extends an observation made by Bousfield in Bou99.

Theorem 4.5. Let $L$ be any localization. Then the following hold:

(i) If $E$ is a connective ring spectrum and $L E$ is connective, then the spectrum $L E$ has a unique ring structure such that the localization map $l_{E}: E \rightarrow L E$ is a ring map. If $E$ is commutative, then $L E$ is also commutative.

(ii) If $M$ is an $E$-module, where $E$ is a connective ring spectrum, then $L M$ has a unique E-module structure such that the localization map $l_{M}: M \rightarrow L M$ is an E-module map. Moreover, if $L E$ is connective, then $L M$ also admits a unique LE-module structure extending the E-module structure.

Proof. Using that $E$ is a connective spectrum, we have equivalences

$$
F^{c}\left(E, F^{c}(X, Y)\right) \simeq F^{c}(E, F(X, Y)) \simeq F^{c}(E \wedge X, Y)
$$

that give a bijection $\left[E, F^{c}(X, Y)\right] \cong[E \wedge X, Y]$. Then one proceeds as in the proof of Theorem 4.2

Remark 4.6. The assumption that $L E$ is connective in part (i) of Theorem 4.5 is automatically fulfilled if $E, A$ and $B$ are connective, by the following argument: A spectrum $X$ is connective if and only if $P_{S} X \simeq 0$, where $S$ is the sphere spectrum. Therefore, if $A$ and $B$ are connective, then $f: A \rightarrow B$ is a $P_{S}$-equivalence. This implies that every $f$-equivalence is also a $P_{S}$-equivalence, and in particular the localization map $E \rightarrow L E$ is a $P_{S}$-equivalence, from which it follows that $L$ does not change the homotopy groups in negative dimensions.

\section{Localization OF STABLE GEMS}

Using results due to Bousfield Bou99] and Rudyak Rud98, we next show that the stable GEMs are precisely the $H \mathbb{Z}$-modules. Thus, the results in the previous section imply that every $f$-localization sends stable GEMs to stable GEMs. In addition, we will describe all the localizations of the ring spectrum $H \mathbb{Z}$.

Definition 5.1. Let $R$ be an arbitrary ring with unit. A spectrum $E$ is called a stable $R$-GEM if it is equivalent to a wedge of suspensions of Eilenberg-Mac Lane spectra, $E \simeq \bigvee_{k \in \mathbb{Z}} \Sigma^{k} H A_{k}$, where each $A_{k}$ is an $R$-module (hence, each $H A_{k}$ is an $H R$-module spectrum). If $R=\mathbb{Z}$, then stable $\mathbb{Z}$-GEMs are called stable GEMs.

Note that, if $E$ is a ring spectrum, then for every spectrum $X$, the smash product $E \wedge X$ has an $E$-module structure given by $\mu \wedge 1: E \wedge E \wedge X \rightarrow E \wedge X$.

For any ring spectrum $(E, \mu, \eta)$, the triple $(E \wedge-, \eta \wedge 1, \mu \wedge 1)$ is a monad on the stable homotopy category HoS. The algebras over this monad are pairs $(M, m)$, where $M$ is a spectrum and $m: E \wedge M \rightarrow M$ is a map that renders certain diagrams commutative; see [Bor94]. These diagrams endow $M$ precisely with the structure of an $E$-module spectrum. Thus, the category of algebras (also called the EilenbergMoore category) associated with the monad $(E \wedge-, \eta \wedge 1, \mu \wedge 1)$ is the category 
whose objects are the $E$-module spectra and whose morphisms are the $E$-module maps in HoS. We denote this category by $\operatorname{HoS}_{E-\bmod }$ and denote by $[M, N]_{E \text {-mod }}$ the corresponding group of $E$-module maps from $M$ to $N$.

Every monad factors as a pair of adjoint functors through its Eilenberg-Moore category; see [Bor94]. In our case, this gives the following result. We are indebted to Gustavo Granja for pointing out this result to us, and to the referee for observing that it comes from the fact that $\operatorname{HoS}_{E \text {-mod }}$ is an Eilenberg-Moore category, so we may omit the proof.

Lemma 5.2. Let $E$ be any ring spectrum. Then the functor $\mathrm{HoS} \rightarrow \mathrm{HoS}_{E-\bmod }$ assigning to each spectrum $X$ the spectrum $E \wedge X$ is left adjoint to the forgetful functor $\mathrm{HoS}_{E \text {-mod }} \rightarrow \mathrm{HoS}$. That is, for every spectrum $X$ and every E-module $M$, there is a natural isomorphism

$$
[X, M] \cong[E \wedge X, M]_{E-\bmod }
$$

induced by the unit of $E$.

Proposition 5.3. For every $H \mathbb{Z}$-module $M$, there is a map of $H \mathbb{Z}$-modules

$$
H \mathbb{Z} \wedge\left(\bigvee_{k \in \mathbb{Z}} \Sigma^{k} M G_{k}\right) \stackrel{\tilde{\alpha}}{\longrightarrow} M
$$

which is an equivalence, where $G_{k}=\pi_{k}(M)$.

Proof. An argument was sketched in Ada74 p. 307]. For each $k \in \mathbb{Z}$, take one map $\alpha_{k} \in\left[\Sigma^{k} M G_{k}, M\right]$ mapping to the identity in $\operatorname{Hom}\left(G_{k}, G_{k}\right)$. Let $\alpha=\bigvee_{k \in \mathbb{Z}} \alpha_{k}$ be the wedge of all $\alpha_{k}$ constructed in this way. This yields, by Lemma 5.2, an $H \mathbb{Z}$-module map

$$
\tilde{\alpha}: H \mathbb{Z} \wedge\left(\bigvee_{k \in \mathbb{Z}} \Sigma^{k} M G_{k}\right) \longrightarrow M
$$

namely $\tilde{\alpha}=m \circ(1 \wedge \alpha)$. Now the map $\tilde{\alpha}$ induces an isomorphism on $\pi_{k}$ for all $k \in \mathbb{Z}$, because $\eta \wedge 1$ and $1 \wedge \alpha$ are isomorphisms on $\pi_{k}$. So $\tilde{\alpha}$ is an equivalence.

Proposition 5.3 tells us that $H \mathbb{Z}$-module spectra and stable GEMs are the same thing, because $H \mathbb{Z} \wedge M G \simeq H G$ for any abelian group $G$, and therefore, if $M$ is an $H \mathbb{Z}$-module, then $M \simeq \bigvee_{k \in \mathbb{Z}} \sum^{k} H \pi_{k}(M)$. Similarly, the $H R$-modules are precisely the stable $R$-GEMs, because each $H R$-module spectrum is an $H \mathbb{Z}$-module spectrum, and the homotopy groups of $H R$-module spectra are $R$-modules (see Remark 4.11).

Corollary 5.4. Let $A$ and $B$ be abelian groups. Then

$$
\begin{aligned}
& {[H A, H B]_{H \mathbb{Z}-\bmod } \cong \operatorname{Hom}(A, B),} \\
& {[H A, \Sigma H B]_{H \mathbb{Z}-\bmod } \cong \operatorname{Ext}(A, B),} \\
& {\left[H A, \Sigma^{k} H B\right]_{H \mathbb{Z}-\bmod }=0 \quad \text { if } k \neq 0,1 .}
\end{aligned}
$$

Proof. As a special case of Proposition 5.3. $H A \simeq H \mathbb{Z} \wedge M A$ as $H \mathbb{Z}$-modules. By Lemma 5.2. there is a natural bijection

$$
\left[M A, \Sigma^{k} H B\right] \cong\left[H A, \Sigma^{k} H B\right]_{H \mathbb{Z}-\bmod },
$$

and the result follows using Lemma 3.2 . 
In the rest of this section, $L$ denotes $f$-localization with respect to a fixed but arbitrary map $f$. Using the results on $f$-localizations of $E$-modules of Section 4 we have the following.

Theorem 5.5. Let $R$ be any ring with unit. If $E$ is a stable $R$-GEM, then $L E$ is a stable $R$-GEM and the localization map $l_{E}: E \rightarrow L E$ is an $H R$-module map.

Proof. A stable $R$-GEM is the same as an $H R$-module, and $H R$ is a connective spectrum. Hence we may apply Theorem 4.5 .

Next, we are going to study the case when the spectrum $E$ is an iterated suspension of a single Eilenberg-Mac Lane spectrum, i.e., $E \simeq \Sigma^{n} H G$, where $G$ is an $R$-module and $n \in \mathbb{Z}$. By Theorem [5.5, we know that $L \Sigma^{n} H G \simeq \bigvee_{k \in \mathbb{Z}} \Sigma^{k} H G_{k}$ with each $G_{k}$ an $R$-module. In fact, most of the $R$-modules $G_{k}$ are zero, as we next explain. Consider the following sequence of $H \mathbb{Z}$-module maps, where $\beta$ is a homotopy inverse of the map given by Proposition 5.3 , and $p_{i}$ is the projection onto the $i$-th factor:

$$
\Sigma^{n} H G \stackrel{l}{\longrightarrow} L \Sigma^{n} H G \stackrel{\beta}{\longrightarrow} \bigvee_{k \in \mathbb{Z}} \Sigma^{k} H G_{k} \stackrel{p_{i}}{\longrightarrow} \Sigma^{i} H G_{i} .
$$

By Corollary 5.4,

$$
\left[\Sigma^{n} H G, \Sigma^{i} H G_{i}\right]_{H \mathbb{Z}-\bmod }=0
$$

unless $i=n$ or $i=n+1$. The universal property of localization and the fact that $\Sigma^{i} H G_{i}$ is $f$-local because it is a retract of $\bigvee_{k \in \mathbb{Z}} \Sigma^{k} H G_{k}$ (see Lemma 2.1) tell us that $G_{i}=0$ if $i \neq n$ or $i \neq n+1$.

Therefore, the localization of any iterated suspension of an Eilenberg-Mac Lane spectrum has at most two nonzero homotopy groups:

Theorem 5.6. Let $G$ be any abelian group and $n \in \mathbb{Z}$. Then

$$
L \Sigma^{n} H G \simeq \Sigma^{n} H G_{1} \vee \Sigma^{n+1} H G_{2}
$$

as $H \mathbb{Z}$-modules, for some abelian groups $G_{1}, G_{2}$. If $G$ is an $R$-module for some ring $R$, then $G_{1}$ and $G_{2}$ are also $R$-modules.

Corollary 5.7. If $E \simeq \bigvee_{i \in \mathbb{Z}} \Sigma^{i} H A_{i}$ then $L E \simeq \bigvee_{i \in \mathbb{Z}} L \Sigma^{i} H A_{i}$

Proof. The spectrum $\bigvee_{i \in \mathbb{Z}} L \Sigma^{i} H A_{i}$ is $f$-local because it is equivalent to the product $\prod_{i \in \mathbb{Z}} L \Sigma^{i} H A_{i}$, by Proposition III.3.14 in [Ada74] (here we use Theorem 5.6 to ensure that, for each value of $i$, only a finite number of homotopy groups - in fact, at most two - are nonzero), and the map

$$
\bigvee_{i \in \mathbb{Z}} \Sigma^{i} H A_{i} \longrightarrow \bigvee_{i \in \mathbb{Z}} L \Sigma^{i} H A_{i}
$$

is an $f$-equivalence, because it is a wedge of $f$-equivalences.

There are some special cases in which the localization of an Eilenberg-Mac Lane spectrum is a single Eilenberg-Mac Lane spectrum.

Theorem 5.8. If $G$ is free abelian and $n$ is any integer, then $L \Sigma^{n} H G \simeq \sum^{n} H A$ for some abelian group A. 
Proof. From Theorem [5.6, we know that $L \Sigma^{n} H G$ has at most two nonzero homotopy groups, say $A$ and $B$. By Corollary 5.4,

$$
\left[\Sigma^{n} H G, \Sigma^{n+1} H B\right]_{H \mathbb{Z}-\bmod } \cong \operatorname{Ext}(G, B) .
$$

If $G$ is free, then $\operatorname{Ext}(G, B)=0$, and this tells us that the projection $\Sigma^{n} H G \rightarrow$ $\Sigma^{n+1} H B$ is nullhomotopic. Moreover, $\Sigma^{n+1} H B$ is $f$-local, because it is a retract of $L \Sigma^{n} H G$. Then the universal property of localization forces that $B=0$.

Theorem 5.9. If $p$ is a prime, $k$ is a positive integer and $n$ is any integer, then $L \Sigma^{n} H \mathbb{Z} / p^{k} \simeq \Sigma^{n} H \mathbb{Z} / p^{i}$ with $0 \leq i \leq k$.

Proof. We assume that $n=0$ for simplicity, and start by considering the case $k=1$. By Theorem 5.6,

$$
L H \mathbb{Z} / p \simeq H A_{1} \vee \Sigma H A_{2},
$$

where $A_{1}$ and $A_{2}$ are $\mathbb{Z} / p$-modules. Since retracts and desuspensions of $f$-local spectra are $f$-local, it follows that either $A_{1}=0$ and $A_{2}=0$, or $H \mathbb{Z} / p$ is $f$-local. If $A_{1}=0$ and $A_{2}=0$, then $L H \mathbb{Z} / p$ is contractible and the cofibre sequences

$$
H \mathbb{Z} / p \longrightarrow H \mathbb{Z} / p^{r} \longrightarrow H \mathbb{Z} / p^{r-1}
$$

imply inductively that $L H \mathbb{Z} / p^{r}$ is contractible for all $r$, by Proposition 2.6.

Now let $k$ be any positive integer. By the preceding discussion, we may assume that $H \mathbb{Z} / p$ is $f$-local. Using Theorem 5.6 again, we may write

$$
L H \mathbb{Z} / p^{k} \simeq H B_{1} \vee \Sigma H B_{2},
$$

where $B_{1}$ and $B_{2}$ are $\mathbb{Z} / p^{k}$-modules (i.e., abelian groups with an exponent dividing $\left.p^{k}\right)$. Therefore, by [Kap69], $B_{1}$ and $B_{2}$ are direct sums of subgroups isomorphic to $\mathbb{Z} / p^{j}$ with $j \leq k$. If $B_{2}=0$, then $\operatorname{Hom}\left(B_{1}, B_{1}\right) \cong \operatorname{Hom}\left(\mathbb{Z} / p^{k}, B_{1}\right)$, by the universal property of $L$, and this forces $B_{1}$ to consist of only one summand and $\mathbb{Z} / p^{k} \rightarrow B_{1}$ to be surjective (as in Theorem 3.4 in Cas00]). Finally, we suppose that $B_{2}$ is nonzero and will arrive at a contradiction. Let $m$ be the smallest integer such that $\mathbb{Z} / p^{m}$ is a direct summand of $B_{2}$. Then $\Sigma H \mathbb{Z} / p^{m}$ and $H \mathbb{Z} / p^{m}$ are $f$-local. Now the cofibre sequences

$$
H \mathbb{Z} / p^{(r+1) m} \longrightarrow H \mathbb{Z} / p^{r m} \longrightarrow \Sigma H \mathbb{Z} / p^{m}
$$

imply inductively that $H \mathbb{Z} / p^{r m}$ is $f$-local for $r \geq 1$, by Lemma 2.2 Choose $r$ such that $r m>k$ and infer that $H \mathbb{Z} / p^{k}$ is $f$-local, by using downwards the cofibre sequences

$$
H \mathbb{Z} / p^{i-1} \longrightarrow H \mathbb{Z} / p^{i} \longrightarrow H \mathbb{Z} / p .
$$

This is indeed a contradiction.

Corollary 5.10. If $G$ is a finitely generated abelian group and $n$ is any given integer, then $L \Sigma^{n} H G \simeq \Sigma^{n} H A$ for some abelian group $A$.

Proof. This follows from Theorem 5.8 and Theorem 5.9, since $L$ commutes with finite products.

Bousfield has informed us that the conclusion that $L \Sigma^{n} H G$ has at most one nonzero homotopy group holds, more generally, if $G$ is a reduced abelian group; that is, if the only divisible subgroup of $G$ is the trivial subgroup. The argument will not be given in this article. 
If we now project $L \Sigma^{n} H G$ onto the first summand

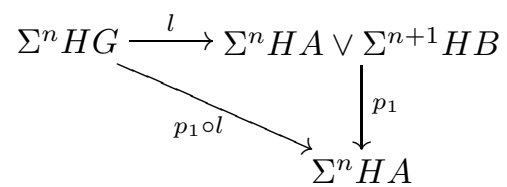

we can obtain information about the group $A$. The universal property of $L$ yields an isomorphism of abelian groups

$$
\left[\Sigma^{n} H A, \Sigma^{n} H A\right] \times\left[\Sigma^{n+1} H B, \Sigma^{n} H A\right] \cong[H G, H A],
$$

where $\left[\Sigma^{n+1} H B, \Sigma^{n} H A\right]=0$ because

$$
[\Sigma H B, H A] \cong(H A)^{0}(\Sigma H B) \cong \operatorname{Hom}\left(\pi_{0}(\Sigma H B), A\right)=0 .
$$

Hence we get

$$
\operatorname{Hom}(A, A) \cong\left[\Sigma^{n} H A, \Sigma^{n} H A\right] \cong[H G, H A] \cong \operatorname{Hom}(G, A) .
$$

In the case when $G=\mathbb{Z}$, this says that $\operatorname{Hom}(A, A) \cong A$. Therefore, if $A$ is nonzero, then $A$ admits a ring structure, with a multiplication coming from composition in $\operatorname{Hom}(A, A)$ and a unit coming from the identity homomorphism. Moreover, the isomorphism $\operatorname{Hom}(A, A) \cong A$ is given by evaluation at the unit.

Definition 5.11. A ring $A$ with unit such that $\operatorname{Hom}(A, A) \cong A$ via $\varphi \mapsto \varphi(1)$ is called rigid.

This terminology was first used in [CRT00. However, rigid rings had previously been studied in a different context, under the name of $E$-rings. The most obvious examples of such rings are $\mathbb{Z}, \mathbb{Q}, \mathbb{Z} / p$, or the $p$-adics $\hat{\mathbb{Z}}_{p}$, for any $p$. There are many other examples: for instance, each product $\prod_{p \in P} \hat{\mathbb{Z}}_{p}$ is rigid, for any set of (distinct) primes $P$. In fact, as shown in DMV87], there are rigid rings of arbitrarily large cardinality. However, neither the group $\mathbb{Z} / p^{\infty}$ of $p$-th roots of unity nor the $p$-adic field $\hat{\mathbb{Q}}_{p}$ admits the structure of a rigid ring. Therefore, there is no localization $L$ whatsoever such that $\pi_{0}(L H \mathbb{Z})$ is isomorphic to $\mathbb{Z} / p^{\infty}$ or $\hat{\mathbb{Q}}_{p}$.

Rigid rings are commutative. Solid rings in the sense of $[\mathrm{BK} 72 \mathrm{~b}]$ are rigid (yet, all solid rings are countable). Proofs of these claims and further details about rigid rings can be found in CRT00.

All rigid rings occur as homotopy groups of localizations of $H \mathbb{Z}$, since, if $A$ is any rigid ring, then $L_{f} H \mathbb{Z} \simeq H A$, where $f$ is the map $H \mathbb{Z} \rightarrow H A$ induced by the unit homomorphism $\mathbb{Z} \rightarrow A$. Alternatively, if we choose $g: S \rightarrow M A$, then the map $H \mathbb{Z} \rightarrow H A$ is a $g$-equivalence, and $H A$ is $g$-local, so $L_{g} H \mathbb{Z} \simeq H A$ as well.

We can summarize the results obtained for $f$-localizations of $H \mathbb{Z}$ in the following theorem.

Theorem 5.12. Let $f$ be any map of spectra. Then the $f$-localization of $H \mathbb{Z}$ has at most one nonzero homotopy group, i.e., $L_{f} H \mathbb{Z} \simeq H A$. Moreover, the group $A$ has a rigid ring structure if $A \neq 0$. All rigid rings appear this way.

Corollary 5.13. There is a proper class of non-equivalent $f$-localizations.

Proof. According to results in DMV87], there is a proper class of non-isomorphic rigid rings. 
We conclude with another application. We say that a localization $L$ is smashing if the natural map $1 \wedge l_{S}: X \rightarrow X \wedge L S$ is an $L$-localization for all spectra $X$, where $l_{S}: S \rightarrow L S$ is the localization of the sphere spectrum. It follows from this definition that every smashing localization is homological (namely, $L \simeq L_{E}$, where $E=L S$ ) and hence it commutes with suspension. Moreover, $L S$ is a commutative ring spectrum, by Theorem 4.2 (this was already observed by Bousfield in [Bou79]), and the multiplication map $L S \wedge L S \rightarrow L S$ is an equivalence, since it is a left inverse of $1 \wedge l_{S}: L S \rightarrow L S \wedge L S$. Conversely, if $E$ is a ring spectrum and the multiplication map $E \wedge E \rightarrow E$ is an equivalence, then $L_{E}$ is smashing, by [Rav84, 1.29].

As also shown in [Rav84, 1.27], a homological localization $L_{E}$ is smashing if and only if it commutes with direct limits. This happens, for example, if $E$ is the spectrum $K$ of (complex) $K$-theory or the Johnson-Wilson spectrum $E(n)$ for any $n$.

Theorem 5.14. If $L$ is smashing, then $H_{n}(L S)=0$ if $n \neq 0$, and it is either zero or a subring of the rationals if $n=0$.

Proof. We have

$$
H_{n}(L S)=\pi_{n}(H \mathbb{Z} \wedge L S) \cong \pi_{n}(L H \mathbb{Z}) \cong \pi_{n}(H A)
$$

for some rigid ring $A$, by Theorem 5.12. Hence, $H_{n}(L S)=0$ if $n \neq 0$. From the fact that $L S \wedge L S \simeq L S$ and the Künneth theorem, we infer that

$$
H_{0}(L S) \otimes H_{0}(L S) \cong H_{0}(L S)
$$

and

$$
\operatorname{Tor}\left(H_{0}(L S), H_{0}(L S)\right)=0 .
$$

Hence, $H_{0}(L S)$ is a torsion-free solid ring. Thus, it is a subring of the rationals, by the classification of solid rings; see [BK72a].

Corollary 5.15. If $L$ is a smashing localization such that $H_{0}(L S) \cong \mathbb{Q}$, then either $L$ is ordinary $H \mathbb{Q}$-localization, or the homotopy groups $\pi_{i}(L S)$ are nonzero for infinitely many negative values of $i$.

Proof. The assumption made implies that $L S \wedge H \mathbb{Q}$ is nonzero, and this implies that $H \mathbb{Q}$ is $L$-local. Now the unit $S \rightarrow H \mathbb{Q}$ factors through $L S$, yielding a map $L S \rightarrow H \mathbb{Q}$ which, according to Theorem 5.14, is an integral homology equivalence. If $L S$ is bounded below, then it follows that $L S \simeq H \mathbb{Q}$, from which we infer that $L X \simeq X \wedge L S \simeq X \wedge H \mathbb{Q}$ for all $X$.

The ring $H_{0}(L S)$ happens to be $\mathbb{Q}$, for instance, if $L$ is localization with respect to $E=K$ or $E=E(n)$ for any $n$. In each of these cases, the spectrum $H \mathbb{Q}$ is $E$-local, since it is a retract of $E \wedge H \mathbb{Q}$ (which is nonzero). Hence, it suffices to show that the natural map $H \mathbb{Z} \rightarrow H \mathbb{Q}$ is an $E$-equivalence. For this, we may use the fact that $E_{k}(H \mathbb{Z})=\lim _{i} E_{k+i}(K(\mathbb{Z}, i))$. For $E=K$, the result follows from [AH68]. For $E=E(n)$, it is a consequence of [Bou82, Example 7.5].

The above remarks yield an easy proof of the well-known fact that the spectra $L_{K} S$ and $L_{E(n)} S$ are not bounded below.

\section{ACKNOWLEDGEMENTS}

Conversations with Gustavo Granja, John Greenlees, Stefan Schwede, Jeff Smith, and Neil Strickland were greatly helpful to us. We are especially indebted to the referee for very pertinent remarks which simplified and improved some of our results. 


\section{ADDED AFTER POSTING}

In the statement of Lemma $2.1, Y$ is meant to be fibrant.

\section{REFERENCES}

[Ada74] J. F. Adams, Stable Homotopy and Generalised Homology, University of Chicago Press, Chicago, 1974. MR53:6534

[AH68] D. W. Anderson and L. Hodgkin, The $K$-theory of Eilenberg-Mac Lane complexes, Topology 7 (1968), pp. 317-329. MR37:6924

[Bad01] B. Badzioch, Recognition principle for generalized Eilenberg-Mac Lane spaces, In: Cohomological Methods in Homotopy Theory (Barcelona, 1998), Progress in Math., vol. 196, Birkhäuser, Basel, 2001, pp. 21-26. MR2002f:55019

[Bor94] F. Borceux, Handbook of Categorical Algebra II: Categories and Structures, Encyclopedia of Mathematics and its Applications, vol. 51, Cambridge University Press, Cambridge, 1994. MR96g:18001b

[Bou77] A. K. Bousfield, Constructions of factorization systems in categories, J. Pure Appl. Algebra 9 (1976/77), no. 2, pp. 207-220. MR57:17648

[Bou79] A. K. Bousfield, The localization of spectra with respect to homology, Topology 18 (1979), no. 4, pp. 257-281. MR.80m:55006

[Bou82] A. K. Bousfield, On homology equivalences and homological localizations of spaces, Amer. J. Math. 104 (1982), no. 5, pp. 1025-1042. MR84g:55014

[Bou96] A. K. Bousfield, Unstable localization and periodicity, In: Algebraic Topology: New Trends in Localization and Periodicity (Sant Feliu, 1994), Progress in Math., vol. 136, Birkhäuser, Basel, 1996, pp. 33-50. MR.98c:55014

[Bou99] A. K. Bousfield, On $K(n)$-equivalences of spaces, In: Homotopy Invariant Algebraic Structures (Baltimore, 1998), Contemp. Math., vol. 239, Amer. Math. Soc., Providence, 1999, pp. 85-89. MR2001k:55013

[BF78] A. K. Bousfield and E. M. Friedlander, Homotopy theory of $\Gamma$-spaces, spectra, and bisimplicial sets, In: Geometric Applications of Homotopy Theory (Evanston, 1977), II, Lecture Notes in Math., vol. 658, Springer-Verlag, Berlin, Heidelberg, New York, 1978, pp. 80-130. MR80e:55021

[BK72a] A. K. Bousfield and D. M. Kan, The core of a ring, J. Pure Appl. Algebra 2 (1972), pp. 73-81. MR 46:7222

[BK72b] A. K. Bousfield and D. M. Kan, Homotopy Limits, Completions and Localizations, Lecture Notes in Math., vol. 304, Springer-Verlag, Berlin Heidelberg New York, 1972. MR.51:1825

[Cas00] C. Casacuberta, On structures preserved by idempotent transformations of groups and homotopy types, In: Crystallographic Groups and Their Generalizations (Kortrijk, 1999), Contemp. Math., vol. 262, Amer. Math. Soc., Providence, 2000, pp. 39-68. MR2001i:55012

[CR97] C. Casacuberta and J. L. Rodríguez, On towers approximating homological localizations, J. London Math. Soc. 56 (1997), pp. 645-656. MR99b:55016

[CRT00] C. Casacuberta, J. L. Rodríguez, and J.-Y. Tai, Localizations of abelian EilenbergMac Lane spaces of finite type, preprint, 2000.

[Dro96] E. Dror Farjoun, Cellular Spaces, Null Spaces and Homotopy Localization, Lecture Notes in Math., vol. 1622, Springer-Verlag, Berlin Heidelberg New York, 1996. MR:98f:55010

[DMV87] M. Dugas, A. Mader, and C. Vinsonhaler, Large E-rings exist, J. Algebra 108 (1987), pp. 88-101. MR 88e:16047

[DP01] W. G. Dwyer, J. H. Palmieri, Ohkawa's theorem: there is a set of Bousfield classes, Proc. Amer. Math. Soc. 129 (2001), no. 3, pp. 881-886. Mr2001f:55015

[EKMM97] A. D. Elmendorf, I. Kriz, M. A. Mandell, and J. P. May, Rings, Modules, and Algebras in Stable Homotopy Theory, Math. Surveys Monographs, vol. 47, Amer. Math. Soc., Providence, 1997. MR97h:55006

[GU71] P. Gabriel and F. Ulmer, Lokal Präsentierbare Kategorien, Lecture Notes in Math., vol. 221, Springer-Verlag, Berlin Heidelberg New York, 1971. MR48:6205 
[GJ99] P. G. Goerss and J. F. Jardine, Simplicial Homotopy Theory, Progress in Math., vol. 174, Birkhäuser, Basel, 1999. MR2001d:55012

[Gut03] J. J. Gutiérrez, Strict modules and homotopy modules in stable homotopy, preprint, 2003.

[Hir03] P. S. Hirschhorn, Model Categories and Their Localizations, Math. Surveys Monographs, vol. 99, Amer. Math. Soc., Providence, 2003. MR2003j:18018

[Hov95] M. Hovey, Cohomological Bousfield classes, J. Pure Appl. Algebra 103 (1995), pp. 45-59. MR.96g:55008

[HPS97] M. Hovey, J. H. Palmieri, and N. P. Strickland, Axiomatic Stable Homotopy Theory, Mem. Amer. Math. Soc. 128 (1997), no. 610. MR98a:55017

[HSS00] M. Hovey, B. Shipley, and J. Smith, Symmetric spectra, J. Amer. Math. Soc. 13 (2000), no. 1, pp. 149-208. MR2000h:55016

[Kap69] I. Kaplansky, Infinite Abelian Groups, The Univ. of Michigan Press, Ann Arbor, 1969. MR38:2208

[Nee01] A. Neeman, Triangulated Categories, Ann. of Math. Studies, vol. 148, Princeton Univ. Press, Princeton, 2001. MR2001k:18010

[Rav84] D. C. Ravenel, Localization with respect to certain periodic homology theories, Amer. J. Math. 106 (1984), pp. 351-414. MR85k:55009

[Rud98] Y. B. Rudyak, On Thom Spectra, Orientability, and Cobordism, Springer Monographs in Math., Springer-Verlag, Berlin Heidelberg New York, 1998. MF99f:55001

[Str04] N. P. Strickland, Axiomatic stable homotopy - a survey, In: Axiomatic, Enriched and Motivic Homotopy Theory (Cambridge, 2002), NATO Science Series II: Mathematics, Physics and Chemistry, vol. 131, Kluwer Academic Publishers, Dordrecht, 2004, pp. 69-98.

Departament d’Àlgebra i Geometria, Universitat de Barcelona, Gran Via, 585, 08007 BARCELONA, SPAin

E-mail address: carles.casacuberta@ub.edu

Departament de Matemàtiques, Universitat Autònoma de Barcelona, 08193, BelLATERRA, SPAin

E-mail address: jgutierr@mat.uab.es

Current address: Departament d'Àlgebra i Geometria, Universitat de Barcelona, Gran Via, 585, 08007 Barcelona, Spain

E-mail address: jgutier@mat.ub.es 